\title{
Sex Differences in Kidney Stone Disease in Chinese Patients with Type 2 Diabetes Mellitus
}

\author{
Ying Xiao ${ }^{a}$ Ling Wei ${ }^{a} \quad$ Xiaofen Xiong ${ }^{a} \quad$ Yuan Yang ${ }^{a} \quad$ Li Li $^{a} \quad$ Ming Yanga \\ Fei Deng ${ }^{b}$ Lin Sun $^{a}$ \\ aDepartment of Nephrology, the Second Xiangya Hospital, Central South University, Changsha, China; \\ b Department of Urology, the Third Xiangya Hospital, Central South University, Changsha, China
}

\section{Keywords}

Kidney stone - Type 2 diabetes mellitus - Risk factors .

Protective factors $\cdot$ Sex differences

\section{Abstract}

Objectives: To investigate the characteristics of kidney stone disease (KSD) among the Chinese population with type 2 diabetes mellitus (T2DM) and identify sex-specific factors associated with KSD. Methods: A single-center, cross-sectional analysis was performed among Chinese patients with T2DM. KSD was identified by ultrasonography or computed tomography results. Demographic data, physical measurements, laboratory measurements, comorbidities, and related medication data were collected and analyzed. Binary logistic regression was used to explore the associated factors. Results: A total of 7,257 patients with T2DM were included in the study, of which $56.1 \%$ were male and $15.0 \%$ were diagnosed with KSD. The male-to-female ratio for KSD among T2DM patients was 1.35. Among all the T2DM patients, male gender, HOMA2-IR, uric acid, and renal cysts were independent risk factors for KSD development, whereas serum phosphorus and the use of angiotensin-converting enzyme inhibitors

\begin{tabular}{ll}
\hline karger@karger.com & ( ) 2020 The Author(s) \\
Published by S. Karger AG, Basel & Karger \\
Targen access & This article is licensed under the Creative Commons Attribution- \\
NonCommercial-NoDerivatives 4.0 International License (CC BY- \\
NC-ND) (http://www.karger.com/Services/OpenAccessLicense). \\
Usage and distribution for commercial purposes as well as any dis- \\
tribution of modified material requires written permission.
\end{tabular}

(ACEls) were independent protective factors for KSD. Among male diabetic patients, triglycerides, HOMA2-IR, renal cysts, and urinary tract infections were all associated with a greater risk of KSD. In contrast, serum phosphorus was associated with a lower risk of KSD. Among female diabetic patients, systolic blood pressure and HOMA2-B were both contributing factors, and ACEls acted as a protective factor for KSD. Conclusion: Among Chinese patients with T2DM, approximately 1 in 7 patients was affected by KSD, and the prevalence was twice as high as that in the general Chinese population. The factors associated with KSD varied by sex among T2DM patients. Focusing on these factors is beneficial for reducing the risk of KSD and delaying kidney damage in diabetic patients.

(c) 2020 The Author(s)

Published by S. Karger AG, Basel

\section{Introduction}

Kidney stone disease (KSD) is a disorder with consistently increasing global prevalence and incidence rates and is thought to be associated with an increased risk for chronic kidney disease (CKD) and end-stage renal dis- 
ease (ESRD) [1]. A review of epidemiological data from seven countries revealed that the prevalence of KSD varied from $1.7 \%$ to $14.8 \%$ [2]. The health care costs associated with KSD have also increased to more than USD 10 billion in 2006 in the United States [3]. In addition, the disparity in the prevalence of KSD by sex is gradually disappearing, although KSD has historically been twice as common in men than in women. A study in the United States revealed that the male-female ratio of KSD decreased from 1.7 in 1997 to 1.3 in 2002, which might be ascribed to lifestyle changes [4]. A recent study in China reported that the age- and sex-adjusted prevalence of KSD was $5.8 \%$ and the male-female ratio was approximately 1.27 (6.5\% in men and 5.1\% in women) [5]. The reason for the increase in KSD among women is not clearly understood and may partly result from lifestyle and dietary changes and increasing obesity and treatment for stones [3].

Diabetes is a common metabolic disease. Type 2 diabetes mellitus (T2DM), which is characterized by insulin resistance (IR), accounts for approximately $90-95 \%$ of diabetes cases. The number of people with diabetes worldwide has increased sharply from 108 million in 1980 to 422 million in 2014. The global age-standardized diabetes prevalence has increased from $4.3 \%$ in 1980 to $9.0 \%$ in 2014 among males and from $5.0 \%$ to $7.9 \%(6.4-9.7)$ among females [6]. In China, the overall prevalence of diabetes was estimated to be $11.6 \%$, with rates of $12.1 \%$ in men and $11.0 \%$ in women [7]. Prior studies have suggested a positive association between diabetes and the risk of kidney stones and this relationship has been highlighted by the effects of IR $[5,8,9]$. Moreover, the severity of T2DM, as measured by glycemic control and IR, is an important risk factor for KSD [10].

Although the epidemiologic correlation between diabetes and KSD has been well documented, clinical characteristics and factors associated with stones in male and female Chinese T2DM patients are still unknown. In this study, we investigated the prevalence of KSD among Chinese T2DM patients retrospectively and assessed the effect of the related factors using data from a single center.

\section{Materials and Methods}

\section{Study Population}

The patients we studied were diagnosed with T2DM in the Endocrinology Department of Second Xiangya Hospital of Central South University from January 2014 to July 2018. The inclusion criteria were as follows: (i) $\geq 18$ years old; (ii) renal and urinary tract ultrasonography or computed tomography results; and (iii) no congenital anomalies in the kidney or urinary tract, no renal tumors, no nephrectomy, no dialysis, and no renal transplantation. The demographic data, physical measurements, laboratory measurements, comorbidities, and related medication data were collected from the electronic medical record system.

\section{Data Collection}

Age, sex, height, weight, blood pressure, diabetic duration, health behaviors (e.g., smoking and alcohol consumption), related medical history (e.g., urinary stones and gout), and self-reported family history of diabetes mellitus were collected in this study. The body mass index (BMI) was calculated as the body mass divided by the body height squared. The waist-hip ratio (WHR) was defined as the waist measurement divided by the hip measurement.

Hemoglobin, albumin, lipid profile (e.g., triglycerides [TG], cholesterol, high-density lipoprotein cholesterol, low-density lipoprotein cholesterol), fasting plasma glucose (GLU), glycated hemoglobin (HbA1c), serum calcium, serum phosphorus, 25-hydroxyvitamin $\mathrm{D}$, uric acid (UA), creatinine, urine $\mathrm{pH}$ (U-pH), 24hour urine volume, and 24-hour urine albumin were also collected. Age (years) was divided into 4 groups according to quartiles (Q1 18-44; Q2 45-59; Q3 60-74; Q4 275). Similarly, serum phosphorus $(\mathrm{mmol} / \mathrm{L})$ was also divided into 4 quartiles $(\mathrm{Q} 1 \leq 0.89$; 220.90 1.02; Q3 1.03-1.15; Q4 21.16) because it did not follow a normal distribution, and the proportion of patients with abnormal serum phosphorus among those we enrolled was only 20.5\% (data not shown). The Homeostasis Model Assessment (HOMA) 2-B and HOMA2-IR, which represent $\beta$-cell function and IR, respectively, were calculated with the HOMA2 calculator (https://www.dtu. ox.ac.uk/homacalculator/, updated 2013).

In addition, we evaluated related comorbidities (e.g., diabetic retinopathy $[\mathrm{DR}]$, diabetic peripheral neuropathy [DPN], diabetic autonomic neuropathy [DAN], diabetic foot ulcer [DFU], diabetic nephropathy [DN], diabetic ketoacidosis, hypertension, coronary heart disease, cerebrovascular disease, fatty liver, renal cysts $[\mathrm{RC}]$, urinary tract infections [UTI]) and use of medication (e.g., glucose-lowering drugs, renin-angiotensin-aldosterone system inhibitors, and lipid-lowering drugs). Additionally, urinary tract ultrasonography and helical computed tomography were performed by well-trained radiologists using the same model of machine in our hospital. Stone characteristics (e.g., length, number, and location) were recorded. When there were multiple kidney stones, the size of the stone was considered as the length of the largest stone.

\section{Statistical Analysis}

Statistical analysis was performed using SPSS statistics software. A normality test was performed before the data were analyzed. Continuous variables with a normal distribution are reported as the mean \pm standard deviation and were compared by Student's $t$ test, and variables with a nonnormal distribution are expressed as the median (interquartile range, 25-75\%) and were analyzed with the Mann-Whitney test. Categorical variables are summarized as counts and percentages, and comparisons were performed by the chi-square test. The reported $p$ values are twotailed, and $p<0.05$ was considered statistically significant. There was no imputation for missing data. The significant variables were included in the logistic regression analysis to evaluate the factors associated with KSD in T2DM patients. The graphs were constructed using GraphPad Prism software. 

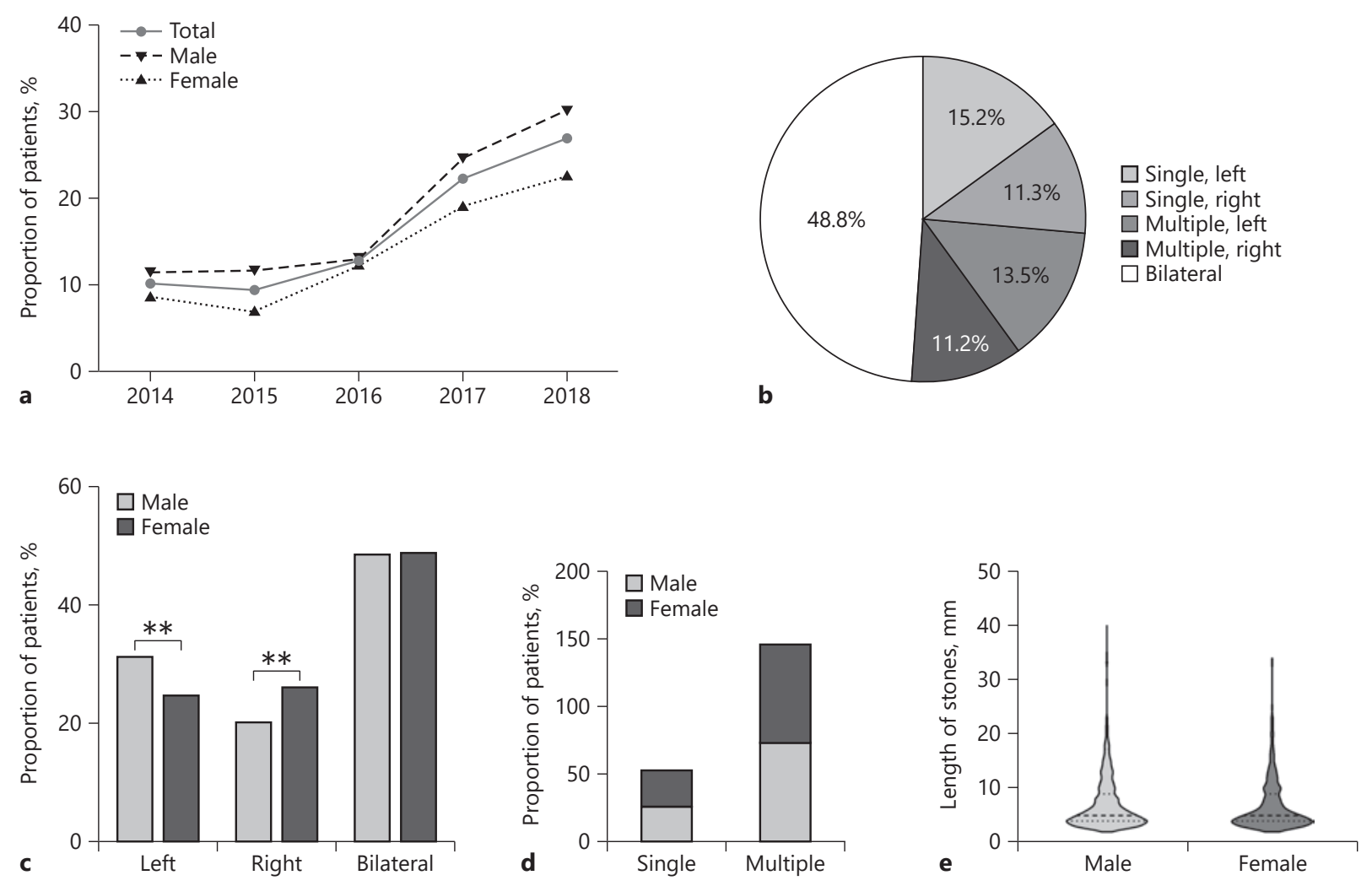

Fig. 1. a Prevalence of KSD among T2DM patients from 2014 to 2018. b Overall distribution of kidney stones in patients with T2DM. c Sex differences in stone locations. d Sex differences in stone numbers. e Sex differences in stone sizes. ${ }^{* *} p<0.01$ versus female group.

\section{Results}

\section{Overview of KSD in Chinese T2DM Patients}

The study included a total of 7,257 patients with T2DM, of which $56.1 \%$ were male and $15.0 \%$ (95\% confidence interval [CI] 14.2-15.8\%) were diagnosed with KSD. The prevalence of KSD was $17.0 \%$ (95\% CI $15.8-$ $18.2 \%$ ) among males and $12.6 \%$ (95\% CI 11.4-13.8\%) among females. In brief, the male-female ratio for KSD in T2DM patients was 1.35. The prevalence of KSD in T2DM patients increased annually, and the increase was more pronounced in males. The prevalence of KSD in 2018 was more than double that in 2014 (Fig. 1a). In terms of the overall distribution of kidney stones, the bilateral distribution accounted for approximately $48.8 \%$ of cases, and patients with a single stone accounted for approximately $26.5 \%$ of patients (Fig. 1b). In addition, in patients with unilateral stones, kidney stones on the left side were more common among men, while stones on the right side were more common among women $(p<0.05)$ (Fig. 1c). However, kidney stones were not significantly varied in number (single or multiple) or size between males and females (Fig. 1d, e). Moreover, GLU, HbAlc, and the proportion of patients with diabetic microvascular complications (DR, DPN, DAN, and DN), which can be related to the severity of diabetes, were not significantly different in diabetic patients with different stone characteristics (length, number, and location) (online suppl. Table S1; see www. karger.com/doi/10.1159/000506053 for all online suppl. material).

\section{Baseline Characteristics of Subjects with/without KSD}

The characteristics of male and female T2DM patients with/without KSD are summarized in Table 1 and online supplementary Table S2. T2DM patients with KSD tended to have a history of urinary stones and more severe IR, as assessed by HOMA2-IR. In terms of laboratory measurements, compared with diabetic patients without stones, pa- 
Table 1. Baseline characteristics of Chinese T2DM patients with/without KSD in both sexes

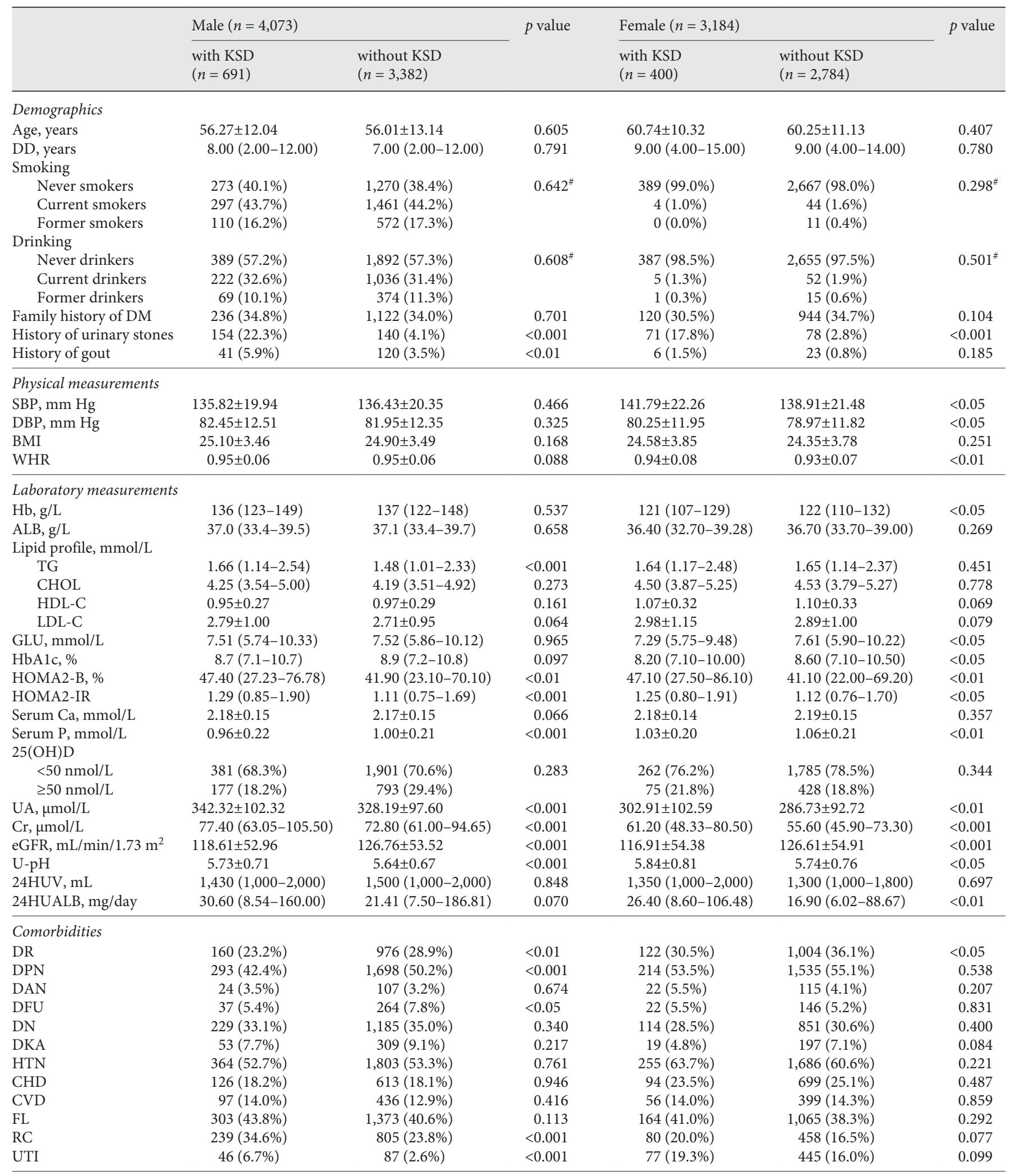


Table 1 (continued)

\begin{tabular}{|c|c|c|c|c|c|c|}
\hline & \multicolumn{2}{|l|}{ Male $(n=4,073)$} & $p$ value & \multicolumn{2}{|c|}{ Female $(n=3,184)$} & $p$ value \\
\hline \multicolumn{7}{|l|}{ Medication use } \\
\hline \multicolumn{7}{|c|}{ Glucose lowering } \\
\hline OHA & $510(73.8 \%)$ & $2,439(72.1 \%)$ & 0.365 & $296(74.0 \%)$ & $2,049(73.6 \%)$ & 0.865 \\
\hline Insulin & $459(66.4 \%)$ & $2,372(70.1 \%)$ & 0.054 & $263(65.8 \%)$ & $1,960(70.4 \%)$ & 0.058 \\
\hline ARBs & $149(21.6 \%)$ & $751(22.2 \%)$ & 0.711 & $133(33.3 \%)$ & $722(25.9 \%)$ & $<0.01$ \\
\hline Lipid lowering & $501(72.5 \%)$ & $2,415(71.4 \%)$ & 0.560 & $300(75.0 \%)$ & $2,050(73.6 \%)$ & 0.562 \\
\hline \multicolumn{7}{|c|}{ 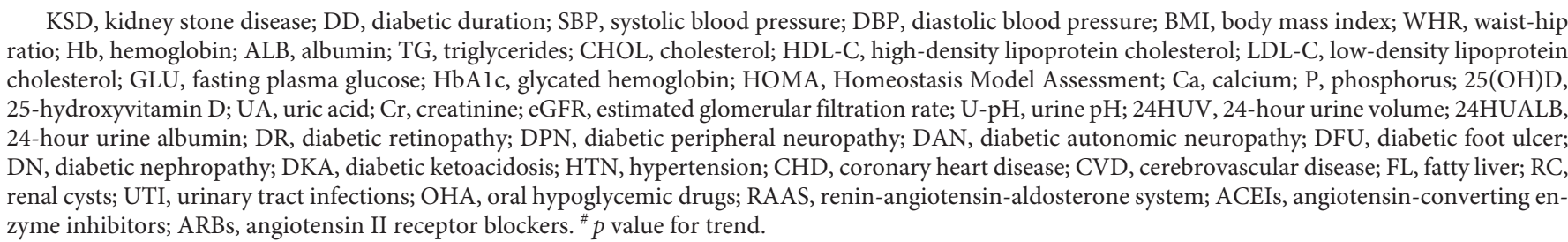 } \\
\hline
\end{tabular}

tients with stones had high serum UA and creatinine levels, higher U-pH but lower serum phosphate levels and estimated glomerular filtration rate (all $p<0.001$ ). Although the $\mathrm{pH}$ was higher in stone formers, it was obvious that both groups had U-pH in the acidic range. In addition, male subjects with KSD were more likely to have a history of gout $(p<0.01)$ and exhibited higher serum TG levels $(p<0.001)$. However, female KSD patients had slightly higher systolic blood pressure (SBP), diastolic blood pressure (both $p<0.05)$, and WHR $(p<0.01)$ than those without kidney stones. Moreover, female subjects with stones showed lower hemoglobin, GLU, and HbAlc (all $p<0.05$ ) but higher 24 -hour urine albumin $(p<0.01)$. Additionally, patients without KSD were prone to $\mathrm{DR}(p<0.001)$. Male patients with stones were less likely to have DPN $(p<$ $0.001)$ and DFU $(p<0.05)$, but had a higher prevalence of RC and UTI (both $p<0.001$ ). In regard to medication, in female patients with KSD, the proportion of angiotensinconverting enzyme inhibitor (ACEI) users was higher than that of nonusers, whereas the ratio of angiotensin II receptor blocker users was much lower (both $p<0.01$ ).

\section{Independent Risk and Protective Factors for KSD in}

T2DM Patients without a History of Urinary Stones

Variables with $p<0.05$ in Table 1 and online supplementary Table S2 were included as covariates in the logistic regression analysis to determine related risk and protective factors for KSD in Chinese T2DM patients without a history of stones or missing data (Fig. 2; see online sup-

Kidney Stone Disease in the Chinese Type 2 Diabetes Population pl. Tables S3-S5). A total of 2,472 male patients and 1,950 female patients were included in the logistic regression models. In the total sample of T2DM patients, male gender (OR 1.22, 95\% CI 1.01-1.48), HOMA2-IR (OR 1.25, 95\% CI 1.04-1.50), UA (OR 1.31, 95\% CI 1.08-1.59), and RC (OR 1.43, 95\% CI 1.16-1.76) were independent risk factors for KSD development, whereas serum phosphorus (2) (Q3 vs. Q1) (OR 0.70, 95\% CI 0.55-0.91), serum phosphorus (3) (Q4 vs. Q1) (OR 0.65, 95\% CI 0.51-0.85), and ACEIs (OR 0.74, 95\% CI 0.58-0.94) were independent protective factors for KSD. In the male diabetic population, TG (OR 1.32, 95\% CI 1.04-1.66), HOMA2-IR (OR $1.30,95 \%$ CI 1.03-1.65), RC (OR 1.56, 95\% CI 1.22-1.99), and UTI (OR 2.30, 95\% CI 1.26-4.19) were all associated with a higher risk for KSD. In contrast, serum phosphorus (2) (OR $0.57,95 \%$ CI $0.41-0.79$ ) and serum phosphorus (3) (OR $0.67,95 \%$ CI $0.48-0.93$ ) were significantly correlated with a lower risk of KSD. In addition, among female diabetic patients, SBP (OR 1.41, 95\% 1.06-1.89) and HOMA2-B (OR 1.96, 95\% CI 1.34-2.86) were both contributing factors, but the use of ACEIs (OR 0.56, 95\% CI $0.37-0.86$ ) could be regarded as a protective factor.

\section{Discussion}

To our knowledge, this is the first study to investigate KSD in a Chinese T2DM population. We found that KSD affected approximately 1 in 7 T2DM patients in China 
Fig. 2. a Factors associated with KSD in the overall sample of Chinese T2DM patients. b Factors associated with KSD in male Chinese T2DM patients. c Factors associated with $\mathrm{KSD}$ in female Chinese T2DM patients. ${ }^{*} p<0.05,{ }^{* *} p<0.01$, and ${ }^{* * *} p<$ 0.001. P, phosphorus.

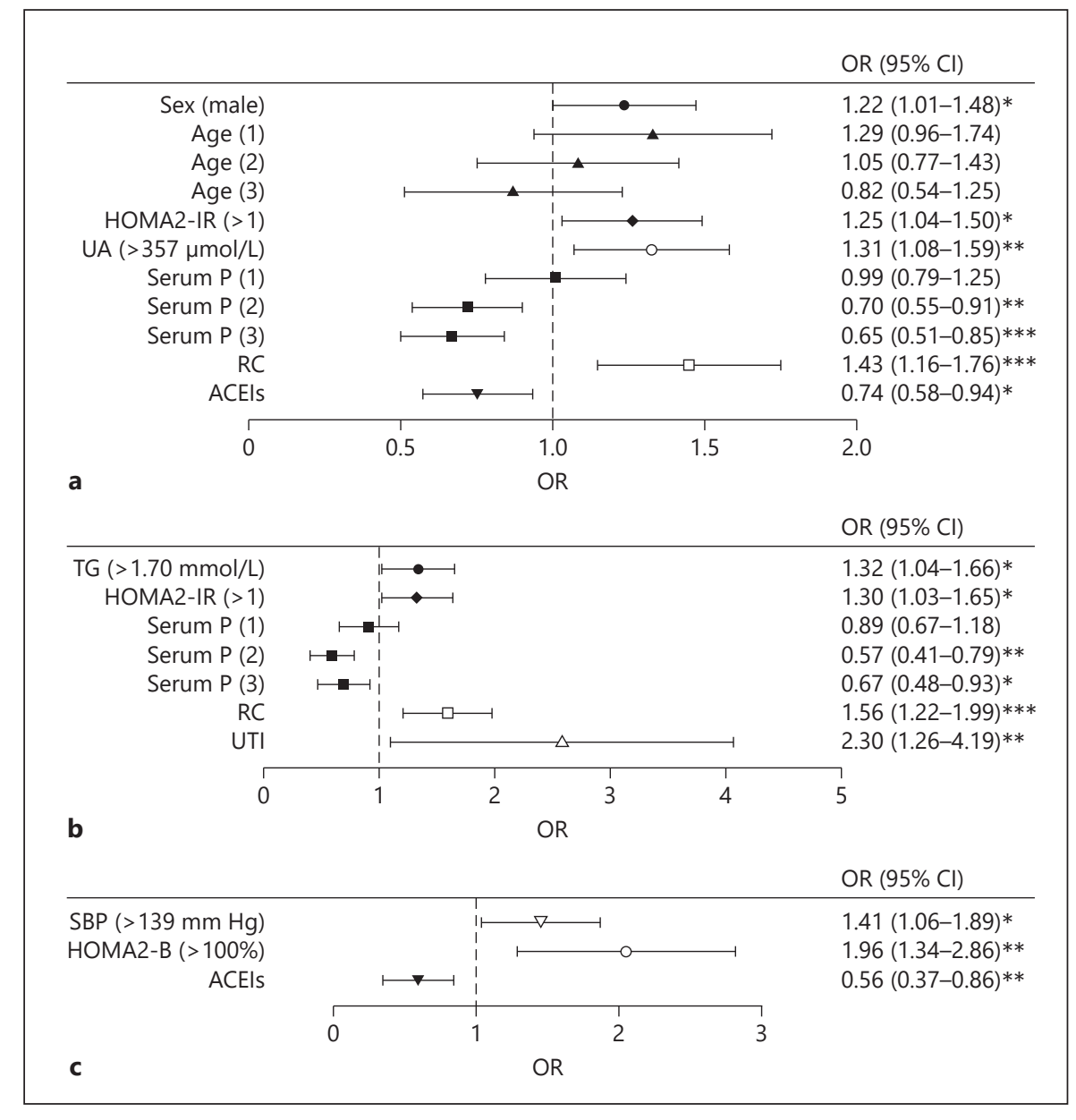

and the male-female ratio for KSD was 1.35. Factors associated with KSD were different by sex. In the male diabetic population, KSD was related to TG, HOMA2-IR, serum phosphorus, RC, and UTI. However, in female diabetic patients, KSD was correlated with SBP, HOMA2$\mathrm{B}$, and the use of ACEIs.

KSD is a common urological disease. In particular, asymptomatic kidney stone formers have a higher risk for ESRD and all-cause mortality [1]. Thus, the reason for progression to ESRD among diabetic patients may include KSD in addition to diabetic kidney disease. Zeng et al. [5] reported that one in 17 adults is currently affected by KSD in China. In the present study, the prevalence of KSD in T2DM patients was twice as high as that in the general Chinese population. One major explanation for this variation might be IR, the core metabolic defect of T2DM, which influences U-pH and reduces the ability of the kidneys to handle ammonium and calcium, thus playing a vital role in stone formation [10]. Canda and Isgoren
[11] also demonstrated that compared to controls, interstitial cells (ICs) and neural tissue were significantly decreased in the bladders of rabbits with diabetes, which might adversely affect the expression and function of ICs in the bladder and urethra, thus affecting ureteral peristalsis and ureteral IC function, ultimately leading to urinary calculi formation. In addition, the male-female ratio for KSD in T2DM patients was similar to that in the general population [5]. This suggests that dietary and lifestyle changes, which are partially responsible for the disparity in sex distribution in the general population, are also involved in female diabetic patients. Therefore, for male and female patients with T2DM, more attention should be paid to kidney stones, and it is necessary to analyze the urine components of these patients so that appropriate treatments can be given to prevent obstructive nephropathy and slow the progression to ESRD.

Metabolic syndrome (MetS) is characterized by the cooccurrence of metabolic abnormalities, including cen- 
tral obesity, dyslipidemia, hypertension, and IR [12]. A systematic review including 355 studies concluded a definite association of MetS with KSD [13], and a cross-sectional study in Japan correlated the number of MetS traits with the severity of KSD [14]. Recently, a prospective cohort in China also suggested that new onset of hypertension, diabetes, and obesity were all strongly associated with an increased risk of kidney stones [15]. In addition, an animal study revealed that the administration of atorvastatin to stone-forming rats significantly lowered crystalline deposits, which may have occurred as a result of its inhibitory effects on renal tubular cell injury and oxidative stress caused by oxalate and crystals [16]. Overall, MetS participates in the pathogenesis of KSD by increasing urinary calcium, $\mathrm{UA}$, and oxalate excretion, as well as decreasing urinary citrate excretion [14]. In the present study, IR and abnormal $\beta$-cell function increased the risk of KSD in male and female T2DM patients, respectively. Moreover, TG increased the risk of KSD in men and SBP increased the risk of stones in women, and TG and SBP are both important MetS traits. The reasons for the sex disparities in risk factors are unclear. However, it is reasonable to speculate that for patients with T2DM, management of MetS traits, especially blood pressure, lipids, IR, and $\beta$-cell function, can not only delay the development of diabetic complications but also prevent stone formation and stone-related complications. Moreover, T2DM patients with these metabolic disorders need to routinely undergo ultrasound examinations to detect stones as early as possible. However, a relationship between obesity (reflected by BMI and WHR) and KSD was not observed, which suggested that obesity may not be a causative factor of stone formation in Chinese T2DM patients, although the correlation between these factors has been concluded in a systematic review [17].

Phosphate overload and eventual hyperphosphatemia are features of $\mathrm{CKD}$, which has emerged as a risk factor for vascular calcification, cardiovascular mortality, and the progression of CKD [18]. To our knowledge, no rigorous studies have proven the relationship between serum phosphorus and stone formation. We observed that serum phosphorus acts as a protective factor for KSD and exerts a maximum protective effect at a relatively high level within the normal range in male diabetic individuals. We speculated that the protective effect of serum phosphorus may be related to calcium and phosphorus homeostasis, although the specific mechanism remains unknown. One notable problem is that some patients were probably in a state of phosphate overload, albeit within normal limits. Fibroblast growth factor 23 (FGF23)

Kidney Stone Disease in the Chinese Type

2 Diabetes Population is increased in the early stages of $\mathrm{CKD}$, even before phosphate retention occurs, and is more suitable to be considered an indicator of phosphate overload [19]. Thus, further studies including the assessment of FGF23 are needed to evaluate the relationship between serum phosphorus and KSD before optimal reference ranges are developed to guide clinical practice. Based on this study, it is safe to conclude that maintaining blood phosphorus levels within the normal range may also be an important measure to prevent KSD in diabetic patients.

RC, especially simple RC, are lesions that are usually benign asymptomatic and are frequently observed in adults [20]. RC are more common in males [21] and are associated with hyperuricemia [22], hypertension [15, 20], and renal dysfunction [23]. RC share some similar characteristics with KSD, but little is known about their specific relationship. We found that male diabetic patients with RC were more likely to suffer from KSD. This finding was in line with a prior study showing that the frequency of kidney stones in the group with RC was twice as high as that in the control group [24]. Genetic and metabolic disturbances in individuals with RC might partially contribute to the increased risk of KSD [25]. Another potential explanation for stone formation in RC patients may be obstruction caused by cysts, albeit it is difficult to tell which caused the obstruction when cysts and stones coexist. In addition, UTI were also strongly correlated with KSD. Increased production of ammonia secondary to infection and the subsequent alkaline urine leads to the formation of stones. Moreover, hypocitraturia further facilitates stone formation [3]. A cohort study revealed that UTI increased the risk of KSD among diabetic patients, and female diabetic patients with UTI tended to be associated with a greater rate of urinary stones [26]. Contrary to this study, we demonstrated that the risk of KSD in male patients with UTI, but not female patients, was 2.3-times higher than that in subjects without UTI. A potential reason for this discrepancy might be geographical and dietary variations in these study populations. T2DM was also associated with an increased risk of UTI [27]. Therefore, for T2DM patients, urine should be checked frequently to exclude the presence of UTI. Patients with RC should receive regular imaging tests, dietary and lifestyle guidance, and appropriate surgical intervention to prevent stone formation and renal damage.

Another topic to discuss is the controversial relationship between ACEIs and KSD. Alexander et al. [28] found that the new prescription of ACEIs was not associated with an altered risk of kidney stone formation. In contrast, Rathod et al. [29] revealed that ACEIs were neg- 
atively correlated with urinary calcium excretion, thus potentially preventing stone formation. This study provided additional evidence that ACEI treatment may lower the risk of KSD. The study by Alexander and his colleagues [28] differs from our study in many ways, including the race of the participants, the inclusion criteria, and the covariates considered. Regarding the observed sex difference, a possible explanation might be the effect of sex hormones. Animal studies have provided evidence that androgens increase urinary calcium excretion by inhibiting the expression of renal calcium transport proteins [30], and estrogen is positively involved in renal calcium reabsorption via the upregulation of epithelial calcium channels [31]. Therefore, it is plausible that androgens may partially reverse the protective effects of ACEIs on calcium excretion and stone formation, although further research is required to test the impact of ACEIs on calcium homeostasis. For male T2DM patients, when using ACEIs, more attention should be paid to stone formation.

Interestingly, sodium-glucose cotransporter2 (SGLT2) inhibitors, a major class of antidiabetic agents, are a double-edged sword in regard to KSD. On the one hand, they may be beneficial for stone prevention by improving IR and $\beta$-cell function and controlling blood pressure. On the other hand, UTI and hypercalciuria are both adverse effects of SGLT2 inhibitors and are associated with a higher risk of KSD [32]. Thus, when taking SGLT2 inhibitors, T2DM patients also need to be regularly assessed for KSD. Data on the use of SGLT2 inhibitors were not available in this study, and their effects on stone formation need to be confirmed in further studies.

It is obvious that both the protective and pathogenic factors associated with KSD were completely different between male and female patients in this study. We speculate that these differences may be related to multiple factors, including sex hormones, genetic differences, lifestyle and dietary differences, etc. For example, an animal study showed that androgens increased urinary calcium excretion, thereby promoting stone formation [30]. Thus, it is reasonable to speculate that in the male diabetic population, the effects of androgens can counteract the protective effects of ACEIs that we observed in females. In other words, androgens partly account for the different effects of ACEIs on stone formation between patients of different sexes. Thus far, although the specific mechanism underlying the sex disparities remains unknown, our novel findings may provide new insights for subsequent clinical and basic studies of KSD.
The results of our study have potential implications for individualized healthcare delivery, particularly for Chinese patients with T2DM. Our findings highlight the importance of metabolic evaluation in diabetic patients, especially lipid profile, blood pressure, and IR. In addition, maintaining serum phosphorus at a relatively high level within the normal range may be beneficial to prevent stone formation in men. Moreover, RC and UTI may be indicators of KSD in male patients. Finally, when using ACEIs, more attention should be paid to stone formation in male patients with T2DM.

Our investigation has important strengths. We analyzed the distribution and clinical characteristics of KSD in the Chinese diabetic population for the first time. The diagnosis of KSD conducted primarily through imaging rather than self-reporting was more credible because it avoids recall bias and detects asymptomatic KSD. The study also had the following limitations. First, the study population was recruited from a single center, which might limit the generalizability of the findings. Second, other potential confounders, including stone composition, dietary information, fluid intake, physical activity, and other related ongoing pharmacological treatments (e.g., thiazide diuretics, vitamin C, vitamin $\mathrm{D}$, and calcium supplements) were unavailable. To clarify the characteristics of KSD among T2DM patients, further prospective, population-based, multicenter studies are needed. The mechanisms underlying the sex disparities, particularly the effects of sex hormones, dietary differences, and genetic predisposition on stone formation, are also worth studying.

In conclusion, KSD affected approximately 1 in 7 T2DM Chinese patients and the prevalence was twice as high as that in the general Chinese population. The risk and protective factors for stones varied by sex among T2DM patients. It is necessary to address these factors in the treatment of patients with diabetes, which may help delay the progression of kidney damage.

\section{Statement of Ethics}

The cross-sectional study was approved by the ethics committee of the Second Xiangya Hospital of Central South University. Formal informed consent is not required. Our institutional board deems the study exempt. This research study was conducted in accordance with the guidelines of the Declaration of Helsinki.

\section{Disclosure Statement}

The authors have no conflicts of interest to declare. 


\section{Funding Sources}

This work was supported by grants from the National Key R\&D Program of China (2018YFC1314002) and the Key Program of General Program of the National Natural Science Foundation of China (NSFC) (81730018).

\section{Author Contributions}

All authors participated in the design of the study. Ying Xiao analyzed the data, interpreted the results, and wrote the manuscript. Ying Xiao, Ling Wei, and Xiaofen Xiong contributed to data collection. Yuan Yang provided support for statistical analysis. Li Li, Ming Yang, and Fei Deng contributed to the revision of the manuscript. Lin Sun is the corresponding author and was involved in the study design, data interpretation, and manuscript revision. All authors read and approved the final manuscript.

\section{References}

1 Dhondup T, Kittanamongkolchai W, Vaughan LE, Mehta RA, Chhina JK, Enders FT, et al. Risk of ESRD and Mortality in Kidney and Bladder Stone Formers. Am J Kidney Dis. 2018 Dec;72(6):790-7.

2 Romero V, Akpinar H, Assimos DG. Kidney stones: a global picture of prevalence, incidence, and associated risk factors. Rev Urol. 2010;12(2-3):e86-96.

3 Khan SR, Pearle MS, Robertson WG, Gambaro G, Canales BK, Doizi S, et al. Kidney stones. Nat Rev Dis Primers. 2016 Feb;2(1): 16008.

4 Scales CD Jr, Curtis LH, Norris RD, Springhart WP, Sur RL, Schulman KA, et al. Changing gender prevalence of stone disease. J Urol. 2007 Mar;177(3):979-82.

5 Zeng G, Mai Z, Xia S, Wang Z, Zhang K, Wang L, et al. Prevalence of kidney stones in China: an ultrasonography based cross-sectional study. BJU Int. 2017 Jul;120(1):109-16.

6 Collaboration NC; NCD Risk Factor Collaboration (NCD-RisC). Worldwide trends in diabetes since 1980: a pooled analysis of 751 population-based studies with 4.4 million participants. Lancet. 2016 Apr;387(10027):1513-30.

$7 \mathrm{Xu} \mathrm{Y,} \mathrm{Wang} \mathrm{L,} \mathrm{He} \mathrm{J,} \mathrm{Bi} \mathrm{Y,} \mathrm{Li} \mathrm{M,} \mathrm{Wang} \mathrm{T,} \mathrm{et}$ al.; 2010 China Noncommunicable Disease Surveillance Group. Prevalence and control of diabetes in Chinese adults. JAMA. 2013 Sep;310(9):948-59.

8 Taylor EN, Stampfer MJ, Curhan GC. Diabetes mellitus and the risk of nephrolithiasis. Kidney Int. 2005 Sep;68(3):1230-5.

9 Aune D, Mahamat-Saleh Y, Norat T, Riboli E. Body fatness, diabetes, physical activity and risk of kidney stones: a systematic review and meta-analysis of cohort studies. Eur J Epidemiol. 2018 Nov;33(11):1033-47.

10 Weinberg AE, Patel CJ, Chertow GM, Leppert JT. Diabetic severity and risk of kidney stone disease. Eur Urol. 2014 Jan;65(1):242-7.

11 Canda AE, Isgoren AE. Re: Increased risk of diabetes in patients with urinary calculi: a 5-year followup study: S.-D. Chung, Y.-K. Chen and H.-C. Lin J Urol 2011; 186: 18881893. J Urol. 2012 Jun;187(6):2279-80.
12 Wei J, Zeng C, Gong QY, Li XX, Lei GH, Yang TB. Associations between Dietary Antioxidant Intake and Metabolic Syndrome. PLoS One. 2015 Jun;10(6):e0130876.

13 Wong Y, Cook P, Roderick P, Somani BK. Metabolic Syndrome and Kidney Stone Disease: A Systematic Review of Literature. J Endourol. 2016 Mar;30(3):246-53.

14 Kohjimoto Y, Sasaki Y, Iguchi M, Matsumura $\mathrm{N}$, Inagaki T, Hara I. Association of metabolic syndrome traits and severity of kidney stones: results from a nationwide survey on urolithiasis in Japan. Am J Kidney Dis. 2013 Jun;61(6):923-9.

15 Ping H, Lu N, Wang M, Lu J, Liu Y, Qiao L, et al. New-onset metabolic risk factors and the incidence of kidney stones: a prospective cohort study. BJU Int. 2019 Dec;124(6):102833.

16 Tsujihata M, Momohara C, Yoshioka I, Tsujimura A, Nonomura N, Okuyama A. Atorvastatin inhibits renal crystal retention in a rat stone forming model. J Urol. 2008 Nov; 180(5):2212-7.

17 Carbone A, Al Salhi Y, Tasca A, Palleschi G, Fuschi A, De Nunzio C, et al. Obesity and kidney stone disease: a systematic review. Minerva Urol Nefrol. 2018 Aug;70(4):393-400.

18 Gonzalez-Parra E, Tunón J, Egido J, Ortiz A. Phosphate: a stealthier killer than previously thought? Cardiovasc Pathol. 2012 Sep-Oct; 21(5):372-81.

19 Rodelo-Haad C, Rodríguez-Ortiz ME, Martin-Malo A, Pendon-Ruiz de Mier MV, Agüera ML, Muñoz-Castañeda JR, et al. Phosphate control in reducing FGF23 levels in hemodialysis patients. PLoS One. 2018 Aug; 13(8):e0201537.

20 Lee YJ, Kim MS, Cho S, Kim SR. Association between simple renal cysts and development of hypertension in healthy middle-aged men. J Hypertens. 2012 Apr;30(4):700-4.

21 Ozveren B, Onganer E, Türkeri LN. Simple Renal Cysts: Prevalence, Associated Risk Factors and Follow-Up in a Health Screening Cohort. Urol J. 2016 Mar;13(1):2569-75.

22 Han Y, Zhang M, Lu J, Zhang L, Han J, Zhao $F$, et al. Hyperuricemia and overexcretion of uric acid increase the risk of simple renal cysts in type 2 diabetes. Sci Rep. 2017 Jun;7(1): 3802 .
23 Choi JD. Clinical characteristics and longterm observation of simple renal cysts in a healthy Korean population. Int Urol Nephrol. 2016 Mar;48(3):319-24.

24 Chang CC, Kuo JY, Chan WL, Chen KK, Chang LS. Prevalence and clinical characteristics of simple renal cyst. J Chin Med Assoc. 2007 Nov;70(11):486-91.

25 Garcia-Nieto V, Negrete-Pedraza F, LopezGarcia M, Luis-Yanes MI. Are simple renal cysts in childhood associated with kidney stones? Nephrourol Mon. 2012;4(4):596-8.

26 Chen HS, Su LT, Lin SZ, Sung FC, Ko MC, Li CY. Increased risk of urinary tract calculi among patients with diabetes mellitus-a population-based cohort study. Urology. 2012 Jan;79(1):86-92.

27 Nichols GA, Brodovicz KG, Kimes TM, Déruaz-Luyet A, Bartels DB. Prevalence and incidence of urinary tract and genital infections among patients with and without type 2 diabetes. J Diabetes Complications. 2017 Nov; 31(11):1587-91.

28 Alexander RT, McArthur E, Jandoc R, Welk B, Hayward JS, Jain AK, et al. Antihypertensive medications and the risk of kidney stones in older adults: a retrospective cohort study. Hypertens Res. 2017 Sep;40(9):837-42.

29 Rathod A, Bonny O, Guessous I, Suter PM, Conen D, Erne P, et al. Association of urinary calcium excretion with serum calcium and vitamin D levels. Clin J Am Soc Nephrol. 2015 Mar;10(3):452-62.

30 Hsu YJ, Dimke H, Schoeber JP, Hsu SC, Lin $\mathrm{SH}$, Chu $\mathrm{P}$, et al. Testosterone increases urinary calcium excretion and inhibits expression of renal calcium transport proteins. Kidney Int. 2010 Apr;77(7):601-8.

31 Van Abel M, Hoenderop JG, Dardenne O, St Arnaud R, Van Os CH, Van Leeuwen HJ, et al. 1,25-dihydroxyvitamin $\mathrm{D}$ (3)-independent stimulatory effect of estrogen on the expression of $\mathrm{ECaCl}$ in the kidney. J Am Soc Nephrol. 2002 Aug;13(8):2102-9.

$32 \mathrm{Hu} \mathrm{C}$, Jia W. Therapeutic medications against diabetes: what we have and what we expect. Adv Drug Deliv Rev. 2019 Jan;139:3-15. 